\title{
Application of serum pepsinogen and carbohydrate antigen 72-4 (CA72-4) combined with gastrin-17 (G-17) detection in the screening, diagnosis, and evaluation of early gastric cancer
}

\author{
Zengbao Lin ${ }^{1}$, Huiqin Bian ${ }^{1}$, Chaoyuan Chen ${ }^{1}$, Wenling Chen ${ }^{2}$, Qiong Li ${ }^{3}$ \\ ${ }^{1}$ Department of Digestive Endoscopy, the Affiliated People's Hospital of Fujian University of Traditional Chinese Medicine, Fujian, China; \\ ${ }^{2}$ Department of Traditional Medicine, the Affiliated People's Hospital of Fujian University of Traditional Chinese Medicine, Fujian, China; \\ ${ }^{3}$ Department of Preventive Treatment, the Affiliated People's Hospital of Fujian University of Traditional Chinese Medicine, Fujian, China \\ Contributions: (I) Conception and design: Z Lin, Q Li; (II) Administrative support: H Bian; (III) Provision of study materials or patients: Z Lin, H \\ Bian, W Chen, Q Li; (IV) Collection and assembly of data: All authors; (V) Data analysis and interpretation: H Bian, C Chen, Q Li; (VI) Manuscript \\ writing: All authors; (VII) Final approval of manuscript: All authors. \\ Correspondence to: Qiong Li. Department of Digestive Endoscopy, the Affiliated People's Hospital of Fujian University of Traditional Chinese \\ Medicine, No. 602, 817 Middle Road, Taijiang District, Fujian 350004, China. Email: qq29113101@126.com.
}

Background: Gastric cancer is a common malignant tumor. The aim of the present study was to analyze the application value of serum pepsinogen (PG), carbohydrate antigen 72-4 (CA72-4), and gastrin-17 (G-17) detection in the screening, diagnosis, and evaluation of early gastric cancer.

Methods: In total, 122 patients with gastric cancer treated in our hospital from January 2018 to January 2021 were selected as the gastric cancer group and subdivided into the early gastric cancer (group A) and advanced gastric cancer (group B) groups. Sixty-five patients with benign gastric disease treated in the same hospital during the same period were selected as the control group, and 122 healthy people who underwent physical examination during the same period were allocated to the control group. The differences in the levels of G-17, PGI, PGII, PGI/PGII, and CA72-4 were compared; receiver-operating characteristic curves were drawn; and the efficacy of different factors in the diagnosis of early gastric cancer was calculated.

Results: G-17, PGI, and PGI/PGII levels in the gastric cancer group were significantly lower than those in the healthy group, and CA72-4 was significantly higher than that in the healthy group $(\mathrm{P}<0.05)$, but there was no significant difference in PGII between the 2 groups $(\mathrm{P}>0.05)$. G-17, PGI, and PGI/PGII levels in groups A and B were significantly lower than those in the control group. CA72-4 in groups A and B was significantly higher than that of the control group, and was highest in group $\mathrm{B}(\mathrm{P}<0.05)$. The areas under the curve (AUC) of G-17, PGI, PGI/PGII, and CA72-4 were 0.671, 0.726, 0.769, and 0.602, respectively, and the AUC of combined detection was 0.883 , which was significantly higher than that of single detection.

Conclusions: Serum PG, CA72-4 combined with G-17 detection has high sensitivity and specificity in the screening and diagnosis of early gastric cancer, and has high clinical application value.

Keywords: Serum pepsinogen (serum PG); carbohydrate antigen 72-4 (CA72-4); gastrin-17 (G-17); gastric cancer screening; early gastric cancer

Submitted Apr 16, 2021. Accepted for publication May 28, 2021.

doi: 10.21037/jgo-21-254

View this article at: http://dx.doi.org/10.21037/jgo-21-254

(C) Journal of Gastrointestinal Oncology. All rights reserved. 


\section{Introduction}

Gastric cancer is a common malignant tumor of the digestive tract, with the mortality rate ranking the second among all malignant tumors, and the incidence rate increasing annually (1). Due to unspecific patient symptoms during the early stage, gastric cancer is often misdiagnosed, causing delays in patient treatment and affecting the treatment effect. There are a number of studies reporting on the detection of serum indicators of gastric cancer (2); However, the sensitivity and specificity vary. The detection of some indicators can be used for the accurate diagnosis of gastric cancer at the early or advanced stage and further instruct the targeted treatment of gastric cancer (2). Some studies have proposed that the combined detection of some appropriate tumor markers can effectively improve the accuracy of diagnosis, thereby improving the prognosis of patients (3). Both gastrin-17 (G-17) and serum pepsinogen (PG) are secreted by gastric mucosa cells, and G-17 is believed to be related to the growth and metastasis of gastric cancer cells. PG is a precursor of pepsin, and some studies have shown that the low serum PGI level and low PGI/ PGII ratio are related to the occurrence and development of gastric cancer (4). Sugar carbohydrate antigen 72-4 (CA72-4), as a special marker for the diagnosis of gastric cancer, has also been reported in many literatures (5). However, there are few studies on the value of the combined detection of three markers in the early screening and diagnosis of gastric cancer. Therefore, in the present study, gastric cancer patients admitted to the Affiliated People's Hospital of Fujian University of Traditional Chinese Medicine from January 2018 to January 2021 were used as the research participants. We studied the application of serum PG and CA72-4, combined with G-17 screening, in gastric cancer patients and healthy controls. The aim of the present study was to analyze the application value of these 3 indicators in the screening, diagnosis, and evaluation of early gastric cancer. We present the following article in accordance with the STARD reporting checklist (available at http://dx.doi.org/10.21037/jgo-21-254).

\section{Methods}

\section{General information}

A total of 122 gastric cancer patients treated in our hospital from January 2018 to January 2021 were selected as the gastric cancer group. Inclusion criteria were as follows: (I) all patients were confirmed to have gastric cancer by gastroscopy and pathological examination; (II) patients who were not receiving medication or chemotherapy; and (III) patients and their family members agreed to participate in the present study, and informed consent was received. Exclusion criteria were as follows: (I) patients with a history of gastric surgery; (II) patients who had taken drugs, such as proton pump inhibitors and gastric protectants within 2 weeks before this treatment; and (III) patients who had coagulation dysfunction or other diseases that affected this study. According to the differentiation degree of gastroscopy, the 122 patients in the gastric cancer group were subdivided into the early gastric cancer group (group A, postoperative pathologically confirmed lesions not exceeding the submucosa) and advanced gastric cancer group (group B). There were 61 cases in each group. In group A, there were 35 males and 26 females, aged 30 70 years old, with an average age of $46.25 \pm 6.54$ years, and with a body mass index (BMI) of $22.36 \pm 2.18 \mathrm{~kg} / \mathrm{m}^{2}$. In group B, there were 33 males and 28 females, aged 30 70 years old, with an average age of $46.28 \pm 6.42$ years, and with a BMI of $22.44 \pm 2.31 \mathrm{~kg} / \mathrm{m}^{2}$.

In addition, 65 patients with benign gastric diseases treated in the same hospital during the same period were selected as the control group. All patients in the control group were diagnosed with superficial gastritis, duodenal ulcer, or other benign gastric diseases by gastroscopy and clinical manifestations, without any other influences to the study disease. There were 38 males and 27 females in the control group, aged 30-70 years old, with an average age of $46.31 \pm 6.19$ years, and a BMI of $22.35 \pm 2.47 \mathrm{~kg} / \mathrm{m}^{2}$. Finally, 60 healthy people were selected as the normal group after undergoing physical examination during the same period, including 31 males and 29 females, aged 30 70 years old, with an average age of $46.30 \pm 6.16$ years, and a BMI of $22.40 \pm 2.51 \mathrm{~kg} / \mathrm{m}^{2}$, and with no gastric disease or other disease affecting the results of this study. There was no significant difference in general data of the selected research participants between the groups $(\mathrm{P}>0.05$, Table 1). All procedures performed in this study involving human participants were in accordance with the Declaration of Helsinki (as revised in 2013). This study was approved by the Affiliated People's Hospital of Fujian University of Traditional Chinese Medicine (No. 2020-013-02). The informed consent was taken from all the patients.

\section{G-17, PG, and CA72-4 detection methods}

A total of $5 \mathrm{~mL}$ of venous blood was taken from each 
Table 1 Comparison of general data of selected objects

\begin{tabular}{lcccc}
\hline Group & Case & Gender (male/female) & Average age $($ year $)$ & $\mathrm{BIM}\left(\mathrm{kg} / \mathrm{m}^{2}\right)$ \\
\hline Group A & 61 & $35 / 26$ & $46.25 \pm 6.54$ & $22.36 \pm 2.18$ \\
Group B & 61 & $33 / 28$ & $46.28 \pm 6.42$ & $22.44 \pm 2.31$ \\
Control group & 65 & $38 / 27$ & $46.31 \pm 6.19$ & $22.35 \pm 2.47$ \\
Normal group & 60 & $31 / 29$ & $46.30 \pm 6.16$ & $22.40 \pm 2.51$ \\
\hline
\end{tabular}

Table 2 Comparison of G-17, PGI, PGII, PGI/PGII, and CA72-4 levels between the gastric cancer group and control group $(\bar{x} \pm$ S)

\begin{tabular}{lcccccc}
\hline Group & Case & G-17 (pmol/L) & CA72-4 (IU/mL) & PGI (ng/mL) & PGII (ng/mL) & PGI/PGII \\
\hline Gastric cancer group & 122 & $10.65 \pm 3.57$ & $8.65 \pm 2.40$ & $28.52 \pm 8.22$ & $25.14 \pm 2.63$ & $2.62 \pm 0.86$ \\
Control group & 122 & $20.35 \pm 4.11$ & $3.22 \pm 0.18$ & $76.35 \pm 10.31$ & $24.68 \pm 2.77$ & $4.63 \pm 1.44$ \\
$t$ & - & 19.680 & 24.988 & 40.065 & 1.33 & 13.236 \\
P value & - & $<0.001$ & $<0.001$ & $<0.001$ & 0.184 & $<0.001$ \\
\hline
\end{tabular}

CA72-4, carbohydrate antigen 72-4; G-17, gastrin-17; PG, pepsinogen.

research subject in the early morning, then centrifuged to obtain the supernatant for further detection. Enzymelinked immunoassay was used to detect the levels of G-17, PGI, PGII, PGI/PGII, and CA72-4 using the Automatic biochemical analyzer (Abbott, Chicago, USA) and the ARCHITECT12000 automatic immunoassay analyzer and assistant supporting reagents (Abbott, Chicago, USA).

\section{Observation indicators}

Differences in the levels of G-17, PGI, PGII, PGI/PGII, and CA72-4 among the different groups were compared.

The value of detection of different factors in the diagnosis of early gastric cancer was then analyzed.

\section{Statistical analysis}

Statistical analysis was performed using SPSS version 18.0 software (SPSS, Chicago, IL, USA). Count data were expressed as $n(\%)$ and analyzed using $\chi^{2}$-test. Measurement data were expressed as $\bar{x} \pm \mathrm{S}$ and analyzed by Student's $t$-test for the comparison of 2 groups, and by F-test among multiple groups. Receiver-operating characteristic curve (ROC) was used for the analysis of the diagnostic sensitivity, specificity and area under curve (AUC) of G-17, PG, CA724 , and the combined detection of the 3 indicators for gastric cancer. Results with $\mathrm{P}<0.05$ was considered statistically significant.

\section{Results}

Comparison of G-17, PGI, PGII, PGI/PGII, and CA72-4 levels between the gastric cancer and control groups

G-17, PGI, and PGI/PGII levels in the gastric cancer group were significantly lower than those in the healthy group $(\mathrm{P}<0.05)$, while the level of CA72-4 was significantly higher than that in the healthy group $(\mathrm{P}<0.05)$. There was no significant difference in PGII level between the 2 groups (P>0.05) (Table 2).

\section{Comparison of G-17, PG, and CA72-4 levels in patients with gastrointestinal diseases}

G-17, PGI, and PGI/PGII levels in groups A and B were significantly lower than those in the control group. The level of G-17 of group A was the lowest among these groups, and the levels of PGI and PGI/PGII in group B were lowest $(\mathrm{P}<0.05)$. The level of $\mathrm{CA} 72-4$ in groups $\mathrm{A}$ and $\mathrm{B}$ was significantly higher than that in the control group and was highest in group $\mathrm{B}(\mathrm{P}<0.05)$. There was no significant difference in PGII levels among the 3 groups $(\mathrm{P}>0.05)$ (Table 3).

Analysis of the value of G-17, PGI, PGI/PGII, and CA724 in the diagnosis of gastric cancer

The ROC results showed that the areas under the curve 
Table 3 Comparison of G-17, PGI, PGII, PGI/PGII, and CA72-4 levels in patients with digestive tract diseases $(\bar{x} \pm \mathrm{S})$

\begin{tabular}{lcccccc}
\hline Group & Case & G-17 $(\mathrm{pmol} / \mathrm{L})$ & CA72-4 $(\mathrm{IU} / \mathrm{mL})$ & $\mathrm{PGI}(\mathrm{ng} / \mathrm{mL})$ & $\mathrm{PGIl}(\mathrm{ng} / \mathrm{mL})$ & $\mathrm{PGI} / \mathrm{PGII}$ \\
\hline Group A & 61 & $3.55 \pm 1.20$ & $4.31 \pm 1.63$ & $33.68 \pm 6.57$ & $25.36 \pm 5.14$ & $2.61 \pm 1.50$ \\
Group B & 61 & $13.68 \pm 2.11^{\mathrm{a}}$ & $9.68 \pm 2.64^{\mathrm{a}}$ & $25.64 \pm 6.54^{\mathrm{a}}$ & $26.81 \pm 5.37$ & $1.33 \pm 0.98^{\mathrm{a}}$ \\
Control group & 65 & $15.64 \pm 3.54^{\mathrm{ab}}$ & $2.87 \pm 1.17^{\mathrm{ab}}$ & $40.65 \pm 8.47^{\mathrm{ab}}$ & $25.32 \pm 3.47$ & $4.84 \pm 1.37^{\mathrm{ab}}$ \\
F & - & 418.22 & 214.38 & 68.48 & 1.96 & 113.50 \\
P value & - & $<0.001$ & $<0.001$ & $<0.001$ & 0.143 & $<0.001$ \\
\hline
\end{tabular}

a , compared with group $\mathrm{A}, \mathrm{P}<0.05 ;{ }^{\mathrm{b}}$, compared with group $\mathrm{B}, \mathrm{P}<0.05$. CA72-4, carbohydrate antigen 72-4; G-17, gastrin-17; PG, pepsinogen.

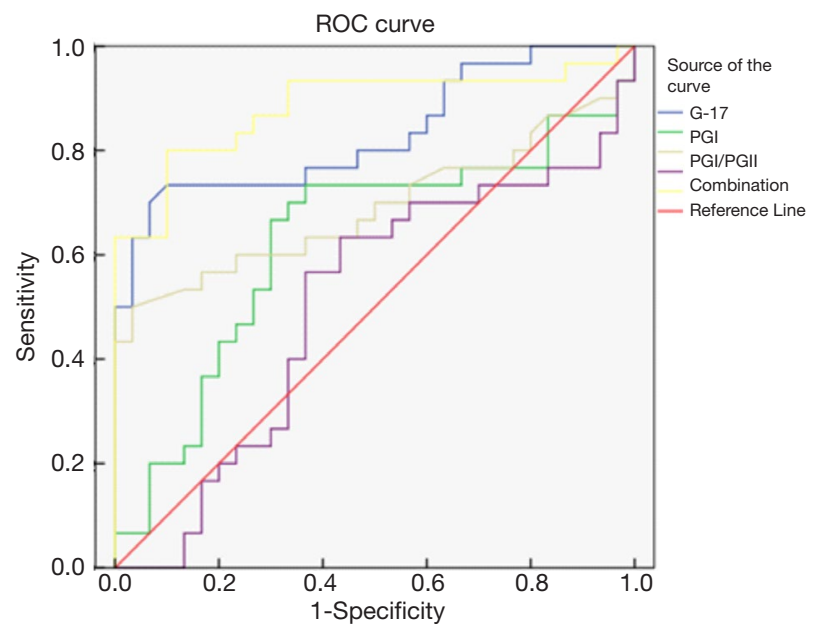

Figure 1 ROC curves of G-17, PGI, PGI/PGII, and CA724 in single and combined detection. ROC, receiver-operating characteristic; G-17, gastrin-17; PG, pepsinogen; CA72-4, carbohydrate antigen 72-4.

(AUC) of G-17, PGI, PGI/PGII, and CA72-4 were 0.671, $0.726,0.769$, and 0.602 , respectively. The AUC of the combined detection was 0.883 , which was significantly higher than that of the single detection of each indicator (Figure 1 and Table 4).

\section{Discussion}

Gastric cancer can be divided into early and advanced, according to its pathological characteristics. The lesions of early gastric cancer are limited to the mucosal layer or submucosa, and early-stage gastric cancer often has a good prognosis. The 5-year survival rate of early gastric cancer is as high as $90 \%$, while the 5 -year survival rate of advanced gastric cancer is less than $10 \%(6,7)$. However, the early diagnostic rate of gastric cancer in China is only about $10 \%$, and the low diagnostic rate is an important factor leading to disease progression, poor prognosis, and the reduced survival rate of gastric cancer $(8,9)$. Therefore, selecting the appropriate methods for early screening and diagnosis is important for improving prognosis and the survival rate.

G-17 is a kind of polypeptide hormone that can stimulate the secretion of $90 \%$ gastric acid. When the stomach shows multifocal atrophy, the level of G-17 is obviously downregulated (10). In the present study, the comparison of serum G-17 levels in different populations showed that the G-17 level of patients with gastric cancer was significantly lower than that of the control group, and the G-17 level of patients with early gastric cancer was significantly lower than those with advanced or benign gastric diseases, which might be due to the decrease in G-17 secretion and the increase in the consumption of G-17 at the early stage. Hypergastrinemia in advanced gastric cancer can lead to higher levels of G-17 compared with early gastric cancer $(11,12)$. The results of the ROC curve analysis showed that G-17 had high sensitivity for the diagnosis of early gastric cancer, but the specificity was not high. Combined with previously reported analyses, the level of G-17 may be affected by many factors, such as eating habit, gastric lesion location, drugs, and G-cell number, so in clinical practice, it is not effective to use G-17 alone as a diagnostic reference indicator (13).

PG is a pepsin precursor that secreted by the main cells of the gastric mucosa, and can be transformed into active pepsin through hydrochloric acid and activated pepsin in the stomach. PGI and PGII are the main members of the PG family (14). PG is almost exclusively secreted by the stomach, and the secretion amount will also change during the secretion stage. Therefore, PG subgroups can reflect the 
Table 4 Analysis of the diagnostic value of G-17, PGI, PGI/PGII, and CA72-4 in gastric cancer

\begin{tabular}{llccc}
\hline Predictive factor & AUC & $95 \% \mathrm{Cl}$ & Sensitivity & Specificity \\
\hline G-17 & 0.671 & $0.624 \sim 0.837$ & 0.610 & 0.523 \\
PGI & 0.726 & $0.678 \sim 0.873$ & 0.711 & 0.730 \\
PGI/PGII & 0.769 & $0.658 \sim 0.833$ & 0.702 & 0.766 \\
CA72-4 & 0.602 & $0.651 \sim 0.758$ & 0.689 & 0.610 \\
Combination & 0.883 & $0.791 \sim 0.976$ & 0.791 & 0.810 \\
\hline
\end{tabular}

AUC, area under the curve; CA72-4, carbohydrate antigen 72-4; Cl, confidence interval; G-17, gastrin-17; PG, pepsinogen.

changes in the number of gastric mucosal glands and cells, as well as the secretory function of different mucosal sites $(15,16)$. When the gastric mucosa is atrophic, the number of gastric corpus glands and fundus glands decrease due to the replacement of the pyloric glands, followed by the decrease of principal cells. After the gastric mucosa function impairment, the level of PGI decreases significantly, while the level of PGII remains stable or slightly increased. The increase in PGII level is due to its wide distribution and the regulation of pyloric gland metaplasia, which ultimately leads to a significant decrease in the ratio of PGI/PGII $(17,18)$. Therefore, changes in the level of PGI and the ratio of PGI/PGII in clinical practice can effectively reflect the functional status of the gastric mucosa (19). Previous studies have shown that changes in PGI level and the PGI/PGII ratio can be used as diagnostic markers for atrophic gastritis, and in Japan, PGI $<70 \mathrm{~g} / \mathrm{L}$ and PGI/PGII $<3.0$ have already been used as the threshold for gastric cancer screening (20). In the present study, the level of PGI and the ratio of PGI/ PGII of gastric cancer patients were significantly lower than those of the control group, both of which were the lowest in advanced gastric cancer $(\mathrm{P}<0.05)$. There was no significant difference in PGII levels between the groups.

As a glycoprotein antigen with high molecular weight, GA72-4 is commonly used in the detection of malignant tumors in the stomach, lung, colon, and other tissues in the clinical setting, because it is almost not expressed in normal tissues or exudates of normal tissues. In the present study, the level of GA72-4 in patients with gastric cancer was significantly higher than that of patients in the control group, and the level of advanced gastric cancer was the highest compared to the early gastric cancer group and the control group. However, the results of the ROC curve in the present study and previous clinical studies indicate that CA72-4 does not have high diagnostic value in gastric cancer. The sensitivity and specificity of CA72-4 combined with G-17 detection were $0.791 \%$ and 0.810 in serum PG, which were significantly higher than those of the three tests alone, and the area under the curve of the combined test was 0.883 , which was significantly higher than that of the three tests alone. These results demonstrate that the combined detection of the 3 indicators can improve the sensitivity and specificity of early screening diagnosis for gastric cancer and meet the requirements for early clinical screening better.

In summary, the combined detection of serum PG, CA72-4, and G-17 has high sensitivity and specificity in early gastric cancer screening and diagnosis with quick and simple detection method, which can provide reference for early diagnosis of gastric cancer and screening of high-risk population, and has high clinical value. In clinical practice, the changes of serum PG, CA72-4 and G-17 levels can be monitored, which can provide certain reference information for the changes of patients' conditions.

\section{Acknowledgments}

Funding: None.

\section{Footnote}

Reporting Checklist: The authors have completed the STARD reporting checklist. Available at http://dx.doi.org/10.21037/ jgo-21-254

Data Sharing Statement: Available at http://dx.doi. org/10.21037/jgo-21-254

Conflicts of Interest: All authors have completed the ICMJE uniform disclosure form (available at http://dx.doi. org/10.21037/jgo-21-254). The authors have no conflicts of interest to declare. 
Ethical Statement: The authors are accountable for all aspects of the work in ensuring that questions related to the accuracy or integrity of any part of the work are appropriately investigated and resolved. This study was approved by the Affiliated People's Hospital of Fujian University of Traditional Chinese Medicine (No. 2020013-02). All procedures performed in this study involving human participants were in accordance with the Declaration of Helsinki (as revised in 2013). The informed consent was taken from all the patients.

Open Access Statement: This is an Open Access article distributed in accordance with the Creative Commons Attribution-NonCommercial-NoDerivs 4.0 International License (CC BY-NC-ND 4.0), which permits the noncommercial replication and distribution of the article with the strict proviso that no changes or edits are made and the original work is properly cited (including links to both the formal publication through the relevant DOI and the license). See: https://creativecommons.org/licenses/by-nc-nd/4.0/.

\section{References}

1. Yu G, Wang GX, Wang HG, et al. The value of detecting pepsinogen and gastrin-17 levels in serum for precancerous lesion screening in gastric cancer. Neoplasma 2019;66:637-40.

2. Zagari RM, Rabitti S, Greenwood DC, et al. Systematic review with meta-analysis: diagnostic performance of the combination of pepsinogen, gastrin-17 and antiHelicobacter pylori antibodies serum assays for the diagnosis of atrophic gastritis. Aliment Pharmacol Ther 2017;46:657-67.

3. Kim DH, Song EA, Kim SW, et al. Efficacy of toluidine blue in the diagnosis and screening of oral cancer and pre-cancer: A systematic review and meta-analysis. Clin Otolaryngol 2021;46:23-30.

4. Raafat N, Emam WA, Gharib AF, et al. Assessment of serum aflatoxin B1 levels in neonatal jaundice with glucose-6-phosphate dehydrogenase deficiency: a preliminary study. Mycotoxin Res 2021;37:109-16.

5. Shin KH, Kim HH, Kwon BS, et al. Clinical Usefulness of Cancer Antigen (CA) 125, Human Epididymis 4, and CA72-4 Levels and Risk of Ovarian Malignancy Algorithm Values for Diagnosing Ovarian Tumors in Korean Patients With and Without Endometriosis. Ann Lab Med 2020;40:40-7.

6. Angeloco LRN, Arces de Souza GC, Romão EA, et al.
Association of dietary acid load with serum bicarbonate in chronic kidney disease (CKD) patients. Eur J Clin Nutr 2020;74:69-75.

7. Cho JH, Jeon SR, Jin SY, et al. Analysis of factors associated with recovery of the serum pepsinogen ratio after Helicobacter pylori eradication: a longterm follow-up study in Korea. Scand J Gastroenterol 2019;54:1306-14.

8. Shida A, Fukuyama T, Futawatari N, et al. Cancer/testis antigen, Kita-Kyushu lung cancer antigen- 1 and $\mathrm{ABCD}$ stratification for diagnosing gastric cancers. World J Gastroenterol 2020;26:424-32.

9. Takayama T, Suzuki H, Okada K, et al. Prediction of true Helicobacter pylori-uninfected status using a combination of age, serum antibody and pepsinogen: Logistic regression analysis. PLoS One 2020;15:e240040.

10. Han ML, Liou JM, Ser KH, et al. Changes of serum pepsinogen level and $\mathrm{ABC}$ classification after bariatric surgery. J Formos Med Assoc 2021;120:1377-85.

11. Chiang TH, Chiu SY, Chen SL, et al. Serum Pepsinogen as a Predictor for Gastric Cancer Death: A 16-Year Community-based Cohort Study. J Clin Gastroenterol 2019;53:e186-93.

12. Bakker DS, Ariens LFM, Giovannone B, et al. EASI p-EASI: Predicting disease severity in atopic dermatitis patients treated with dupilumab using a combination of serum biomarkers. Allergy 2020;75:3287-9.

13. Zhou X, Zhu H, Zhu C, et al. Helicobacter pylori Infection and Serum Pepsinogen Level With the Risk of Gastric Precancerous Conditions: A Cross-sectional Study of High-risk Gastric Cancer Population in China. J Clin Gastroenterol 2020. [Epub ahead of print]. doi: 10.1097/ MCG.0000000000001444.

14. Miftahussurur M, Waskito LA, Aftab H, et al. Serum pepsinogens as a gastric cancer and gastritis biomarker in South and Southeast Asian populations. PLoS One 2020;15:e230064.

15. Wang Q, Wu Y, Zhang H, et al. Clinical Value of Serum HE4, CA125, CA72-4, and ROMA Index for Diagnosis of Ovarian Cancer and Prediction of Postoperative Recurrence. Clin Lab 2019;65:58-4.

16. Wang Y, Zhu Z, Liu Z, et al. Diagnostic value of serum pepsinogen I, pepsinogen II, and gastrin-17 levels for population-based screening for early-stage gastric cancer. J Int Med Res 2020;48:300060520914826.

17. Bang CS, Lee JJ, Baik GH. Diagnostic performance of serum pepsinogen assay for the prediction of atrophic gastritis and gastric neoplasms: Protocol for a systematic 
review and meta-analysis. Medicine (Baltimore) 2019;98:e14240.

18. Zeng W, Zhang S, Yang L, et al. Serum miR-1013 p combined with pepsinogen contributes to the early diagnosis of gastric cancer. BMC Med Genet 2020;21:28.

19. Li M, Xue F, Yang J, et al. Correlation between tumor marker CA72-4 and prognosis of patients with gastric cancer: A protocol for systematic review and meta-analysis. Medicine (Baltimore) 2020;99:e23723.

20. Kim YJ, Chung WC. Is serum pepsinogen testing necessary in populationbased screening for gastric cancer? Korean J Intern Med 2020;35:544-6.

(English Language Editor: R. Scott)

Cite this article as: Lin Z, Bian H, Chen C, Chen W, Li Q. Application of serum pepsinogen and carbohydrate antigen 72-4 (CA72-4) combined with gastrin-17 (G-17) detection in the screening, diagnosis, and evaluation of early gastric cancer. J Gastrointest Oncol 2021;12(3):1042-1048. doi: 10.21037/ jgo-21-254 